action of the eyelids or dilution and removal of the bacterial inoculum by tears, were responsible for the benign course of superficial prolapse. Bacteria in excess of those producing an invariable panophthalmitis on deeper inoculation could be introduced without effect into the surface of the prolapse while the latter was covered with a valve-like flap of conjunctiva. Although the position of the surface prolapse implies its close proximity to the vascular choroid, there was no histological evidence that phagocytosis of the inoculated bacteria played any significant part.

\title{
Summary
}

The remarkable resistance to destructive infection of the eyeball observed in cases of traumatic prolapse of the vitreous in man can be paralleled experimentally in rabbits using a highly virulent B.pyocyaneus as test organism. After exclusion of merely mechanical factors there still remains some significant difference in the resistance of the superficial and deeper layers of the vitreous.

My best thanks are due to Sir John Parsons for his continued interest in this work. To Professor C. C. Okell I am especially indebted for many valuable suggestions and for repeated help. Dr. G. R. Cameron has helped me with the histology.

The investigation was carried out in the bacteriological department of University College Medical School and the expenses were defrayed by a grant from the Medical Research Council.

\section{A CASE OF SEVERE SYMPATHETIC INFLAMMATION BROUGHT TO A SUCCESSFUL CONCLUSION}

\author{
BY \\ J. Herbert Fisher \\ LONDON
}

As an encouragement to surgeons confronted with the results of severe sympathetic ophthalmitis the following record of a case of this character should not go unpublished. In 1919 a boy, A.E.F., born in 1908, was using a fork with which to bore a hole in a leather belt, when one of the prongs penetrated his right eye. About four or five weeks after the accident the surgeon in charge of the case removed the injured eye. When the patient left the Nursing Home, the left eye was slightly bloodshot and inflamed; drops were used to it and an ointment for local inunction in the neighbourhood of the orbit. He continued to see his 
surgecn once a month for eighteen months, but the vision had progressively failed, and on April 22, 1921, two years after the accident, he came to me at Moorfields Eye Hospital for the first time. I found the eye very severely damaged by sympathetic inflammation. There was still some deep injection: much gross keratitis punctata, some of the spots of quite fantastic shape, was deposited on the back of the cornea. The tension was $-1 \frac{1}{2}$, and the soft globe was squared by the pressure of the recti muscles. The iris was discoloured and the subject of total posterior synechia. The pupil was completely occluded by exudate of inflammatory origin organised on the lens capsule. The vision was only perception of light.

I admitted him as an In-patient and he remained in hospital for twelve weeks: during this time the eye was treated by applications of heat, atropine was used to it, and in all twelve small injections of N.A.B. and of kharsivan were administered. On May 23, 1921, the tension of the eye was measured with McLean's tonometer as less than 10 .

On July 26,1921 , he was discharged with very little alteration in the condition of the eye: the tension was still very sub-normal, though perhaps a very little improved. The blood count, which at first had shown a considerable excess of large lymphocytes, had approached a little nearer to the normal.

For the next six months the patient used hot bathings, and a drop of atropine once daily to the eye, and took gr. i of hydrargyri c. creta internally, as a powder, twice a day in spells of a fortnight separated by three-week intervals.

For the next five years he obtained his education at the Worcester College for the Blind on sightless methods and using Braille. During this period I saw him occasionally at long intervals.

On September 12, 1922, I noted: "Tension still - , and globe squared: the eye flushes on examination: blood vessels from the atrophic iris extend over the adventitious capsule of organised exudate in which the degenerate lens is enclosed. The projection of light rather uncertain in the nasal part of the field."

On November 12, 1926, I readmitted him to Moorfields Hospital, and decided to make an attempt to recover some vision for him, though I was strongly advised by two of my colleagues, with whom I consulted, to leave matters alone as they adjudged any operative procedure on an eye so grossly disorganised by sympathetic inflammation to be quite hopeless.

At this time the vision was still, of course, only perception of light. The projection was fairly good in the temporal field but uncertain to the nasal side. The anterior chamber was shallow, but I thought the tension of the eye perhaps a little less sub-normal. The blood count was now reported to me as normal. 
On November 13, 1926, I had a general anaesthetic administered and after making the usual cataract section endeavoured to do an iridectomy upwards : this was not attended by much success owing to the total posterior synechia of a very friable and atrophied iris. I next attempted to pass a scoop behind the lens, but was quite unable to accomplish this, being frustrated by the tough organised diaphragm of inflammatory origin which enclosed the lens both "fore and aft." I therefore slit peripherally into this adventitious capsule with the point of the Graefe knife introduced through the section I had made in the limbus, and following its line: on my doing this some grumous semi-solid cheesy-looking material exuded; but I was now able to pass one branch of a capsule forceps into the vitreous chamber, the other into the anterior chamber in front of the lens, and thus to grasp the lens between its teeth. With a sharp jerk I was at once successful in dragging out the lens and the fibrous material in which it was enclosed.

The eye, subsequently, did not resent the violent treatment to which I had subjected it; no unfavourable reaction followed though the upward coloboma closed, and the iris became completely updrawn.

On January 5, 1927, I noted "the conjunctiva flushes readily." No new precipitates: some appearance of a feathered hazy area in the lower part of the cornea suggests calcareous or hyaline degeneration. T. still minus. No reflex from fundus obtainable. V. = perception of light, best to the temporal side.

On May 5, 1927, I thought the projection was rather more reliable. On May 10, having again admitted him as an in-patient, after chloroform had been administered I performed an iridotomy with de Wecker's scissors, and left the eye with a centrally placed artificial pupil, which has permanently persisted and presents an appearance almost completely natural. At the first dressing after this, the final, operation, the patient was able to see a nurse moving at the foot of his bed. He left the hospital on May 28, 1927; he was then able to count fingers at 18 inches, and I could obtain a dull reflex through his artificial pupil.

A year later wearing $+18^{\circ} 0 \mathrm{D}$. spherical he read $6 / 36$, and by holding this lens remote from the eye he read 4 Jaeger. He was leading the life of a sighted man: guiding himself about without difficulty and enjoying cinemas where he could read the captions under the pictures.

On October 21, 1932, I last saw this patient. $\mathrm{He}$ is now a student in his second term at a Theological College, reading for Orders in the Church of England. He swims and rows, and plays ping-pong and billiards. I have warned him against any efforts which might conduce to a detachment of retina. The eye though still squared and of a tension of $-1-1 \frac{1}{2}$, presents a good appearance 


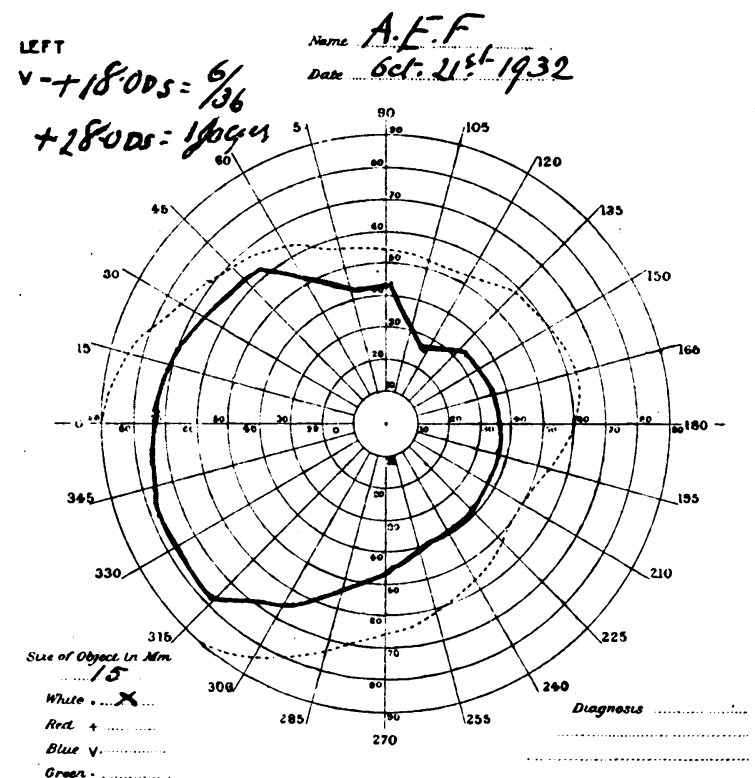

with a central pupil and nothing caught up. With a $+18.0 \mathrm{D}$. spherical he sees $6 / 36$, and with $+28 \cdot 0 \mathrm{D}$. spherical 1 Jaeger fluently, though at a short range: no doubt the soft eye is flattened from before backwards, as well as squared laterally.

Ophthalmoscopic examination is difficult, owing to some corneal degeneration a very little gauzy obstruction in the pupil and some vitreous opacity, but an even red reflex is obtained from all parts of the fundus, and sufficient illumination is obtained to enable me to see dimly the optic disc and vessels in the retina. The very satisfactory field of vision; which is reproduced, was obtained on the perimeter with a $15 \mathrm{~mm}$. test object.

There are a few points which I should like to emphasise :-

The first is, the extreme loyalty of this patient, and the confidence both of himself and of his mother that some day he would recover sight.

The second, that from the outset I had insisted that a long period of years must be allowed to elapse, during which the eye must lie fallow, before any operative procedure should be attempted, if indeed any such would ever be justifiable.

The third, that in the end this eye tolerated the violent interference, which alone could accomplish any useful results, without resentment. Indeed in this respect it proved as hardy as a syphilitic eye, but there was no doubt about the diagnosis and not the slightest suspicion or evidence of syphilis in the case. 